Saily Puentes Bareño*

\title{
La memoria en el proceso de desarrollo y significación de espacios con carácter patrimonial o de ámbito conmemorativo ${ }^{1}$
}

\author{
Memory in the process of development and significance of \\ spaces of type patrimonial or commemorative
}

Cómo citar:

Puentes, S. (2019). La memoria en el proceso de desarrollo y significación de espacios con carácter patrimonial o de ámbito conmemorativo. Designia, 7(1), 65-83.

\author{
${ }^{1}$ Este artículo deriva de la investigación llevada a cabo en el ámbito \\ proyectual presentado en la tesis de pregrado en arquitectura de la \\ Universitaria Agustiniana, titulada: Escenario de construcción colectiva \\ en la Red de la Memoria del centro ampliado del paisaje de Bogotá. \\ *Arquitecta de la Universitaria \\ Agustiniana-Uniagustiniana (Bogotá-Colombia). \\ E-mail: saily.puentes@uniagustiniana.edu.co \\ ORCID: https://orcid.org/0000-0001-9612-411X
}

\section{Palabras clave:}

Diseño arquitectónico, espacio urbano, memoria, signo, paisaje

\section{Key words:}

Architecture design, urban spaces, memory, sign, landscape

\section{Recibido: 31/08/2018}

Aceptado: 28/08/2019

\section{Resumen:}

Este artículo de investigación presenta las premisas en torno al tema de memoria, la cual se compone de una visión acerca de su materialización, del vínculo con la construcción colectiva y de la identidad en el espacio público y los edificios, estos dos últimos como componentes de la arquitectura y el urbanismo que constituyen de forma tangible el soporte para el desarrollo y significación de los espacios, como una forma de narración del conflicto de tierra en el contexto colombiano.

En este sentido, se hace uso de un método de investigación mixto a través de elementos cualitativos y cuantitativos, como puntos claves de estudio para determinar el concepto de la memoria por parte de los usuarios y la población, así como de elementos para la evaluación de la misma en la calidad de vida y su relación con la red digital, empleando tres características para sintetizar las acciones y conclusiones de los hallazgos obtenidos. 


\section{Abstract:}

This research article presents the premises around the theme of Memory, which is composed of a vision about its materialization, the link with collective construction, and identity in public space and buildings, these last two as elements of Architecture and urban planning which have a tangible form of support for the development and significance of spaces, as a way of narrating the land conflict in the Colombian context.

In this sense, a mixed research method is taken through qualitative and quantitative elements, as key points of study to determine the concept of memory by users and population, as well as the elements for the evaluation of it in the quality of life and its relationship with the digital network, using three characteristics to synthesize the actions and conclusions of the findings obtained.

\section{INTRODUCCIÓN}

El lugar de la memoria en la calidad de vida y la reparación simbólica participa a la vez de lo cuantitativo y lo cualitativo, unificado en forma tangible para analizar la realidad del territorio.

Es así que la memoria alude a la noción de la identidad; citando a Souroujon (2011):

... la reconstrucción de la narrativa de la identidad [dota] de sentido, de unidad, a este suceder de sensaciones descubiertas por la memoria; redimiendo el pasado en un todo narrativo que le confiere significación a la existencia, la identidad se contiene en este lazo que desde el presente dota de significación al pasado. (p. 237)

Desde este punto de vista, y frente a este concepto intangible, el lugar cobra sentido y se concreta como escenario.

El contexto se vincula a su vez con la reparación simbólica y la identidad por el interés hacia la memoria colectiva, la cual ha tenido un antecedente principal a partir del Holocausto, de acuerdo a Cabrera (2013), que es cuando ha surgido el derecho a la memoria y la verdad, tanto del interés individual como social en una nación, para evitar la impunidad y el olvido. 
Así, este estudio se inicia con una aproximación de los sucesos y acciones que se han realizado a nivel nacional para resarcir el perjuicio del conflicto, para de esta manera reconocer el proceso de la memoria en ámbitos de reparación y materialización de la misma en el paisaje de Bogotá.

En el país, según la Unidad de Víctimas en Colombia (2018), el conflicto armado sigue vigente con un total de 8708664 personas desplazadas forzadamente y 82 998 desaparecidas, con una raíz común, el conflicto de tierra y la apropiación de los territorios para usos ilícitos, de acuerdo al Centro Nacional de Memoria Histórica (2015).

En este contexto, cabe resaltar en términos cuantitativos que la calidad de vida dada por la cobertura del sistema de salud y vivienda, para la población urbana, ascendió a un déficit del $92 \%$ y $85 \%$, respectivamente, mientras que para las víctimas de desplazamiento forzado en áreas urbanas, estas cifras fueron del 93.2\% y 38.7\%. Esto en relación a una población en Colombia de 47662000 para el año 2014, de acuerdo a la información de las brechas en la calidad de vida de los desplazados en Colombia por parte de la Oficina de Naciones Unidas para la Coordinación de Asuntos Humanitarios (OCHA, 2015).

De forma general, en los párrafos anteriores se presentan algunas de las consecuencias dejadas por el conflicto armado; debido a esto el gobierno, mediante la Ley de Víctimas, o Ley 1448 de 2011, la cual se expide con el objeto de visibilizar y promulgar la reparación integral a las víctimas del conflicto, y para garantizar la no repetición de la guerra, permitió consolidar en todo el territorio leyes y decretos que velen por los derechos de las víctimas urbanas y rurales en el país.

Esta misma ley contiene varios artículos referentes a temas relevantes, como el derecho a la verdad, la reparación simbólica, la restitución de las tierras, entre otros. Para este estudio cabe resaltar el artículo 146, el cual define la creación del Centro de Memoria Histórica, así como las funciones y elementos para el funcionamiento, presupuesto y diseño del Museo Nacional de la Memoria, consignado en el Decreto 4803 de 2011. 
Adicional a esto, paralelamente a la promulgación de la Ley se iniciaron conversaciones con los actores de fuerzas armadas ilegales en torno a la no repetición del conflicto y al papel del Estado para restituir la paz en el país. Como resultado se alcanzó el acuerdo de paz con las FARC (Fuerzas Armadas Revolucionarias de Colombia), el cual se logra luego de diversas fases de diálogo, iniciadas en el año 2012 hasta el 26 de septiembre de 2016, que es cuando se dio en Cartagena la firma final de los acuerdos para la finalización del conflicto, lo cual permitió consolidar un camino para la paz y la reconciliación después de 52 años de guerra (El Tiempo, 2016). Al mismo tiempo, este acto dejaba abierta la posibilidad de diálogo con otros actores del conflicto, como es el caso del ELN (Ejército de Liberación Nacional).

En consonancia con lo anterior, a partir de la reparación simbólica en el proceso de paz, elemento clave que nos atañe como eje para la materialización de la memoria en la calidad de vida, cabe destacar que las poblaciones han habilitado sus propios espacios de conmemoración (parques, placas conmemorativas, esculturas, edificios, salas de exposición), los cuales suman hasta el momento un total de 28 lugares, según la Red Memoria Colombia (2018), de los cuales Bogotá cuenta con un centro de memoria edificado y el lugar del próximo Museo Nacional de la Memoria.

\section{Memoria y materialización}

En lo relacionado con la construcción del Centro de Memoria, Paz y Reconciliación (Indepaz, 2015), la iniciativa a nivel distrital permitió generar una aproximación hacia la reparación y el lugar de la memoria en la ciudad. Esto se consolidaría más tarde con el Decreto 632 de 2014, que dio lugar al proyecto urbano del Eje de la Paz y al diseño del Museo Nacional de la Memoria, el cual, con el objeto de consolidar el espacio público, otorgar reconocimiento a las víctimas y rememorar los hechos recientes en la ciudad y el país, se orienta la integración de los conjuntos patrimoniales a través del eje de la calle 26 o avenida Jorge Eliécer Gaitán, además del predio Ala Solar, lugar de enclave del Museo Nacional de la Memoria, en el paisaje de Bogotá (Secretaria Jurídica Distrital de Bogotá, 2014).

En este contexto, el paisaje hace referencia a la zona ruro-urbana de la ciudad, identificando principalmente el patrimonio edilicio y natural como hito. Aquí, el objeto y lugar tienen sentido con las escalas de actuación (Piñeiro, 2010), como herramienta sistemática para intervenir el espacio a nivel de región y emplearse para materializar la memoria como forma de constructo social (figura 1). 
Territorio Colombiano

Localización ciudad Bogotá

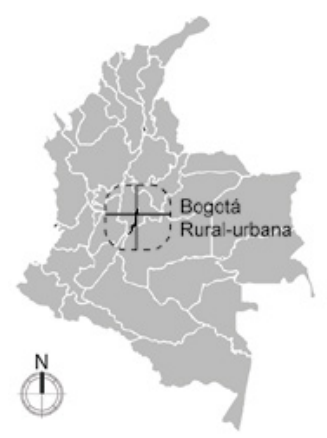

Escala actuación-metropolitana y escala de impacto Bogotá - Centro Ampliado

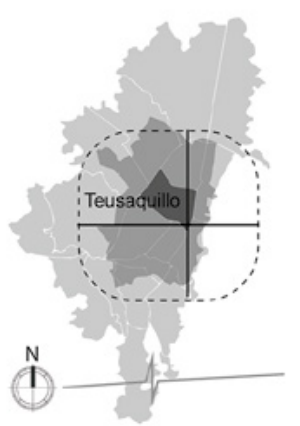

Escala de actuación micro

Predio Ala Solar - Bogotá

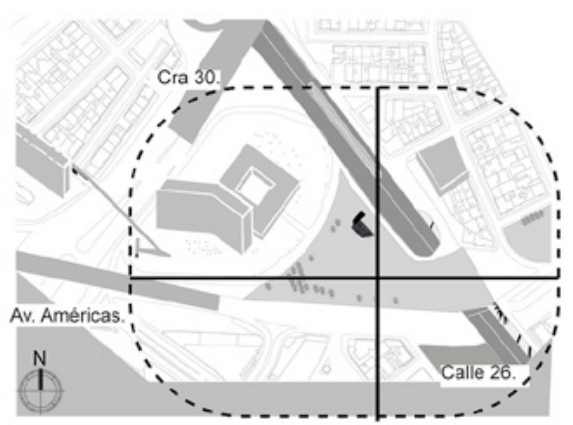

Figura 1. Ubicación geográfica, contexto de reparación simbolica y escalas de actuación.

Fuente: autor.

De esta manera, la ciudad como contenedor de memoria, constituida por los componentes naturales, edificados y la población, resulta vital para la narración y construcción de la identidad, y como espacio para las actividades en el ámbito conmemorativo, tal como lo muestra la figura 2.
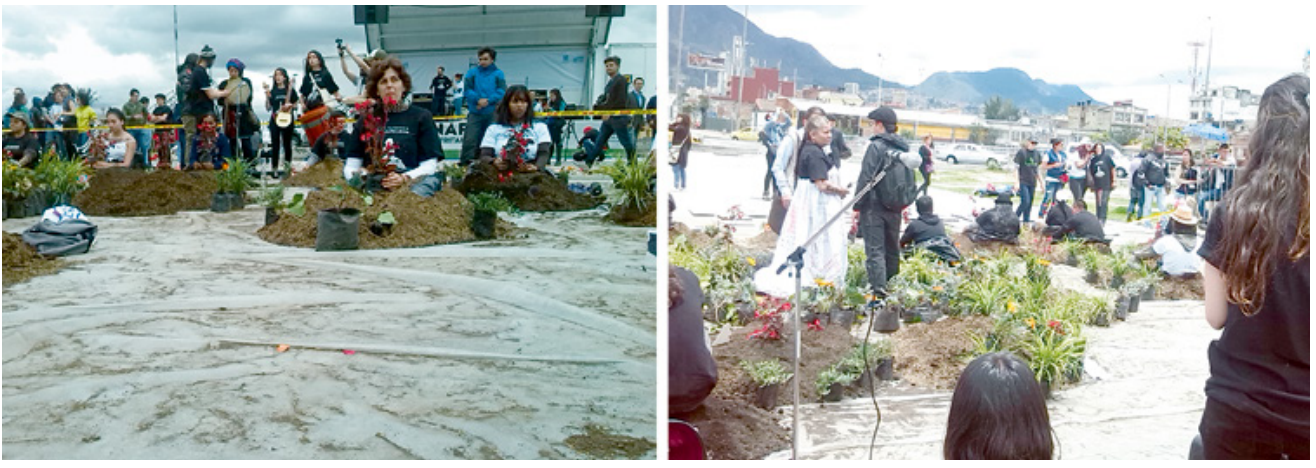

Figura 2. Actividades en torno a la memoria, Día Internacional del Desaparecido, 30 de agosto de 2017, predio Ala Solar, Bogotá.

Fuente: autor. 
En torno a los hechos de paz, y en referencia al lugar y el objeto para la narración de la memoria, la noción de monumento memorial en el ámbito arquitectónico (Velásquez, 2011) surge entonces como espacio para conmemorar y recordar un suceso que no hace parte de la historia principal de la nación, como fundamento para ampliar diferentes eventos y relatos en un periodo de tiempo. Asociado a esto queda el hecho de que el funcionamiento de los memoriales, es decir, su puesta en escena como objeto de divulgación, diálogo y reflexión, transciende a la participación de la población afectada y de los actores en el conflicto para permitir la construcción de las diferentes versiones de los hechos de la guerra.

Es así que desde el conflicto, desde los términos cuantitativos de la calidad de vida en aspectos como vivienda y salud, entre otros, y desde los procesos de reparación simbólica en el país y la importancia del artefacto asociado a ello, hemos introducido el contexto general que antecede a nuestro tema de memoria. En este sentido se refiere a la consolidación de los elementos inmateriales de la población en el lugar (características cualitativas), los cuales se expondrán a lo largo del artículo, para contribuir en el proceso de materialización de los espacios memoriales.

De esta manera, a partir de la pregunta base: ¿Cómo se podrían valorar las categorías de la memoria colectiva en la formulación de proyectos conmemorativos con entornos patrimoniales en el paisaje de Bogotá, y en el ámbito cualitativo de la calidad de vida del púbico visitante y habitante?, se estableció la hipótesis proyectual, la cual responde a la pregunta acerca de cuál tipo de edificio, con su espacio público de soporte, podrá integrar las categorías de la memoria colectiva en el reconocimiento del paisaje, la identidad urbana (patrimonio y cultura) y la calidad de vida del público visitante, los habitantes y la población flotante del paisaje de Bogotá, mediante la formulación y diseño del equipamiento con espacio público en el predio del Ala Solar (Puentes, 2018).

Los procesos que permiten aportar soluciones y ampliar la respuesta al interrogante propuesto se expondrán a través de los siguientes apartados. 


\section{Metodología}

Sistemáticamente, para identificar las características tanto estéticas como sociales del contexto analizado (el área metropolitana, y el memorial en el predio del Ala Solar destinado por el distrito para el desarrollo del Museo Nacional de la Memoria, figura 1), se extrajeron elementos recogidos de los instrumentos y las encuestas, a través de tres etapas principales en la investigación, de modo de sintetizar la metodología empleada con el objeto de obtener los elementos tangibles para la materialización de la memoria.

En la primera etapa, para la construcción de información cualitativa a partir del trabajo de campo y la aplicación de los instrumentos, como encuestas estructuradas y no estructuradas de preguntas cerradas y abiertas, se trabajó en diferentes áreas de la ciudad de Bogotá, con el objeto de obtener datos sobre el significado de la memoria, los recuerdos de la guerra, los materiales, la vegetación y otras características que evocan las regiones del país, empleando el enfoque interpretativo para la transcripción y procesamiento de datos; un ejemplo de esta fase se ilustra en la figura 3.

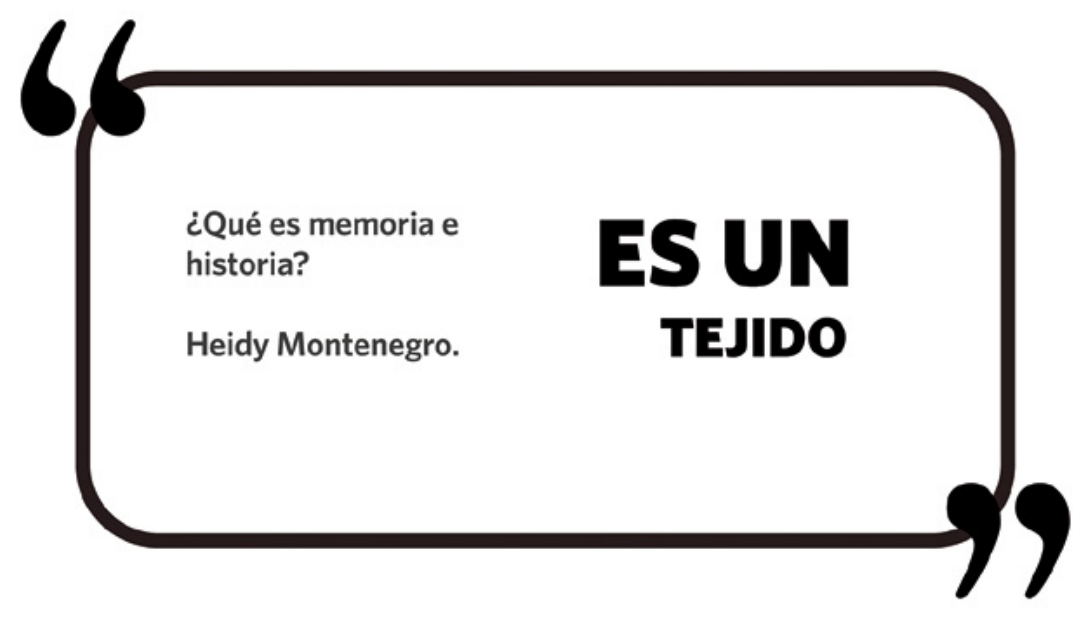

Figura 3. Citas de los usuarios. Encuesta realizada a Heidy Montenegro, 22 de mayo de 2017. 
Paralelo a lo anterior, para extraer los resultados preliminares de las fuentes primarias se realizaron análisis del territorio, permitiendo esbozar el imaginario del lugar, así como obtener datos claves para vincularlos con la segunda etapa de elaboración del marco teórico y conceptual, orientada a la obtención de información de fuentes secundarias.

En la tercera etapa, a partir de los primeros resultados de la investigación se graficaron de manera sistemática los datos, variables y acciones, las cuales se trabajaron en conjunto con las escalas de actuación y el marco teórico, en una síntesis que permitió dar paso al proceso de significación del espacio de carácter patrimonial o de ámbito conmemorativo, de acuerdo a tres factores principales: 1) el usuario, el habitante y actor principal de la narrativa del lugar; 2 ) las dinámicas de los territorios; y 3) el signo relacionado con el paisaje.

\section{Resultados}

En cuanto al planteamiento de conceptos de lugar, significado, identidad, red digital, duelo, calidad de vida y el significado construido de memoria, autores como Echeverría (2009), Jelin (2002), Toledo (2012), Augé (2000) y Leva (2005) toman importancia para identificar en el ámbito proyectual acciones en el territorio, las cuales fueron recopiladas en los factores mencionados para el estudio urbano-arquitectónico:

\section{1) El usuario, habitante y actor principal de la narrativa del lugar}

En esta categoría se identifica a quienes habitan y están presentes en la realidad del suceso, las personas que colaboran en la construcción de la identidad colectiva, de forma que el lugar tiene sentido solo si se habita en él, se utiliza y consolida como propio (Augé, 2000). De tal manera, quien extiende el proceso de la memoria como elemento dentro de la evaluación de la calidad de vida es la población, como se observa en la figura 4. 


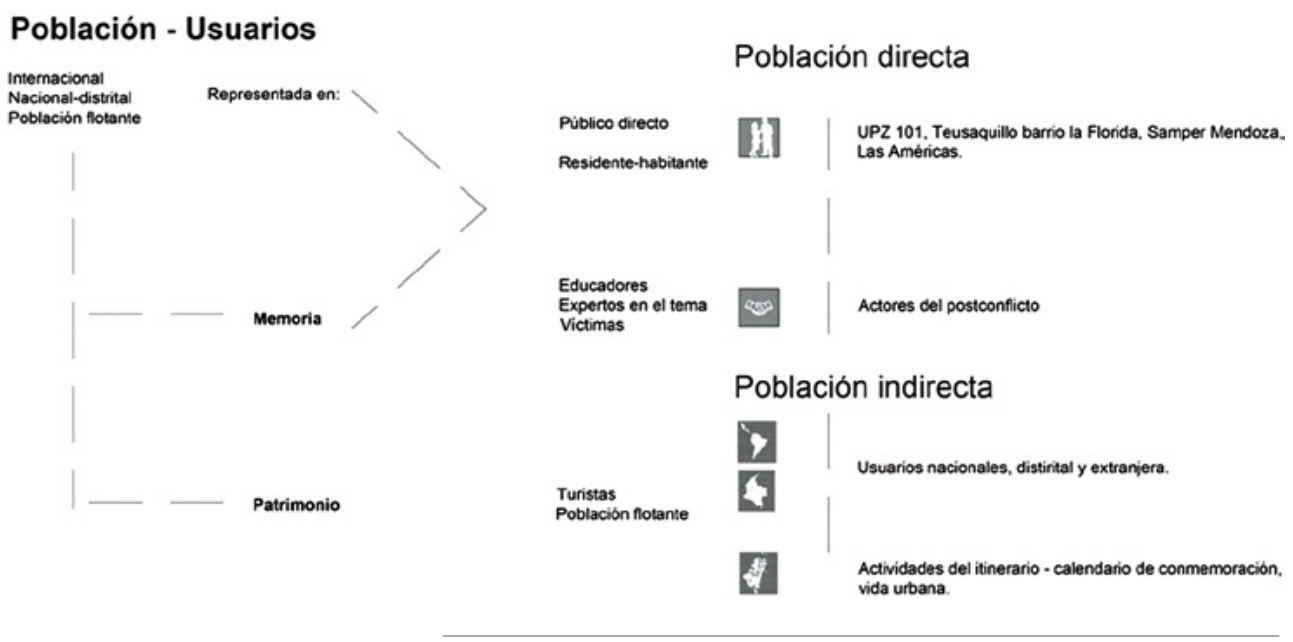

Población directa

Figura 4. Población objeto de estudio (usuarios). Fuente: Elaboración propia a partir de datos del Observatorio Distrital de Víctimas, Unidad para las Víctimas y encuestas realizadas por el autor (2016-2017).

Fuente: autor.

Así, la participación de la población que realiza las narraciones, para construir un concepto de la memoria y acercarse a la valoración de la misma en la calidad de vida de los ambientes de ciudad y locaciones con espacios de conmemoración, se elabora en referencia a la codificación de los elementos cualitativos por parte de los encuestados. 
Esta base cualitativa para definir las categorías de memoria permite el establecimiento de los elementos que convergen en una definición para construir un concepto cercano, propio, que implica un contexto específico y de vivencia, como se observa en la figura 5.

\section{Concepto para la gente acerca de las categorías de memoria histórica + Elementos que identifican la memoria (encuestas).}

\section{Visibilización construcción expandida de la memoria}

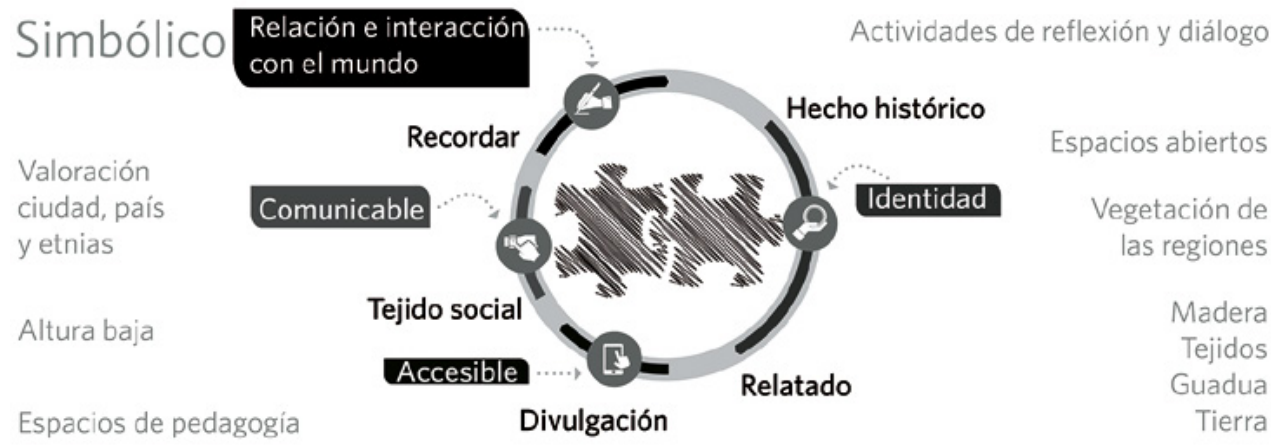

Edificio, actividades de espacio público

Figura 5. Concepto de memoria (valoración de las categorías de la memoria). Fuente: Autor, a partir de las respuestas de los participantes en las encuestas 2016-2017.

De esta manera, los conceptos de memoria, la valoración de la ciudad, el país y las regiones, se integran para construir la segunda característica.

\section{2) Las dinámicas de los territorios}

La variable de la actividad presente en el territorio alude al hecho de contraponer el conflicto de la tierra con el acceso a la misma. De esto cabe resaltar la accesibilidad y el lugar histórico como posibilidad de reflexión y diálogo en torno al concepto de memoria en espacios de carácter público (figura 5). El acceso se compone de permea- 
bilidad y de espacio público, mientras que el lugar, compuesto de las experiencias, la materialidad, la escala y la actividad, constituye lo cercano y próximo para reconocer el contexto (Puentes, 2018).

A partir de aquí, el vínculo del lugar con la memoria nos da indicio de la importancia de la calidad de vida en los procesos de significación territorial, mediante las respuestas de los encuestados en el periodo de 2016-2017, las cuales aportan nuevas alternativas para la medición de la calidad de vida y la valoración de la memoria, como se expone en la figura 6.

INDICADOR

-Acceso: proporción de objetos digitales en puntos estratégicos de la ciudad con acceso público a la memoria digital.

-Narración histórica: nivel de catalogación del hecho histórico, uso de edificios patrimoniales, percepción de evento central para ser narrado.

-Representación-tradiciones: nivel de participación en la elaboración y construcción de una reflexión de la memoria histórica.

-Elementos para recordar: elementos del patrimonio que se identifiquen para restauración y adecuación para la implementación de realidad aumentada para su valoración histórica.
GRUPO

Material y funcional

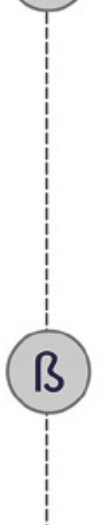

Simbólico

Figura 6. Algunos indicadores de la calidad de vida, con la incorporación de la memoria. Fuente: Elaboración propia a partir de los indicadores de calidad de vida (Leva, 2005). 
Estas categorías colaborarían como referencia de evaluación en los proyectos de los futuros escenarios de memoria.

En consonancia con esto, la relevancia hacia lo simbólico diversifica el ámbito de materialización y su vínculo con lo local. En el caso del ítem $\beta$ de la figura 2, de la representación y las tradiciones surgen expresiones como las actividades, referidas como elementos para recordar en la figura 5.

Desde el ámbito urbano y de edificios, a partir de los instrumentos, se otorga jerarquía a las exposiciones, el duelo y los talleres para la pedagogía. También se contemplan las conmemoraciones y las actividades urbanas para la participación ciudadana, como el Día Nacional de la Solidaridad con las Víctimas del Conflicto cada 9 de abril, el Día del Desaparecido cada 30 de agosto (figura 2), y demás fechas que fomentan la integración de los habitantes en las prácticas, la cultura y saberes en medio del conflicto, para de esta manera colaborar con escenarios para la actividad, enfocados a la construcción constante de la paz (figura 7).

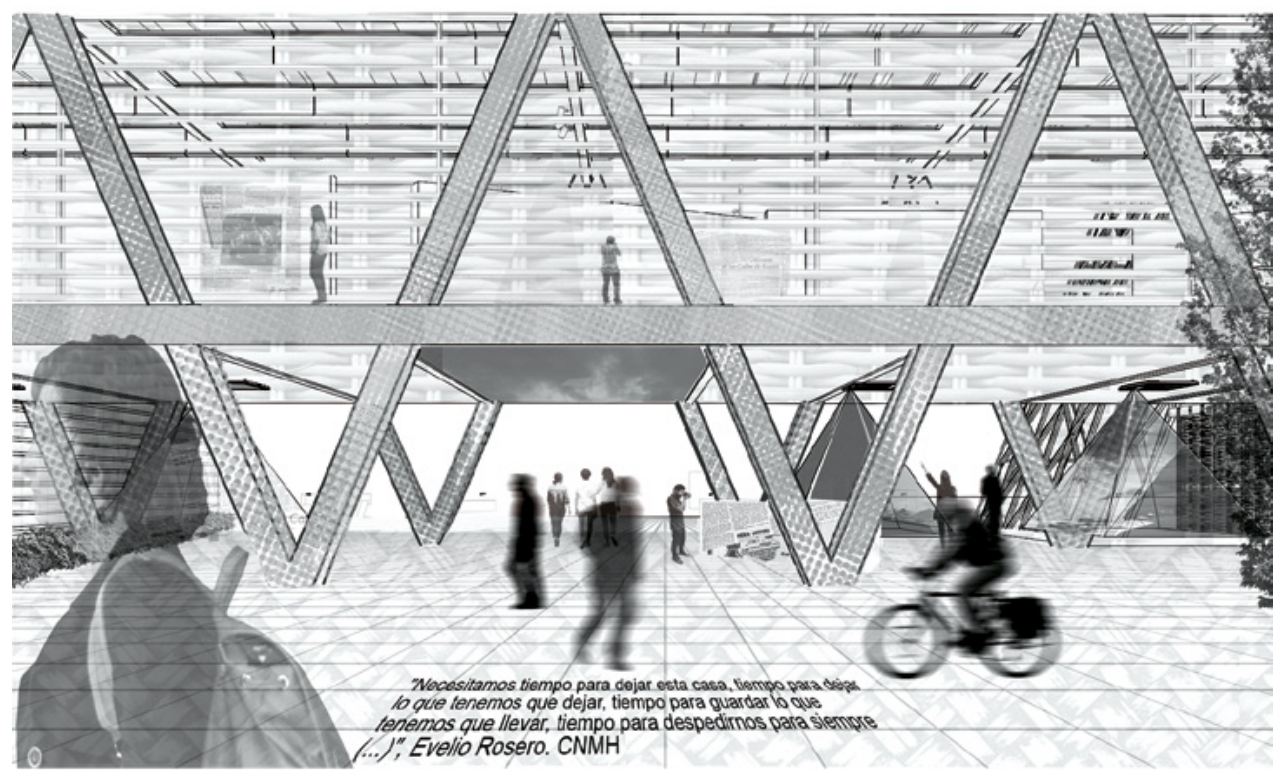

Figura 7. Arcadas, entre lo cercano y la actividad. 
De esta forma se emplean las escalas de actuación para articular los nodos o lugares patrimoniales existentes en la ciudad a nivel de paisaje, como es el caso de los cerros orientales, el parque Bicentenario, los cementerios, la Universidad Nacional, el aeropuerto El Dorado, entre otros, representados en la figura 8. Cabe resaltar que el predio del Museo Nacional de la Memoria se reinterpreta desde el concepto de escenario, correspondiente al sector $\mathrm{C}$ en la figura 8, entre los diversos lugares, y su papel como nodo principal es el de articular la red.

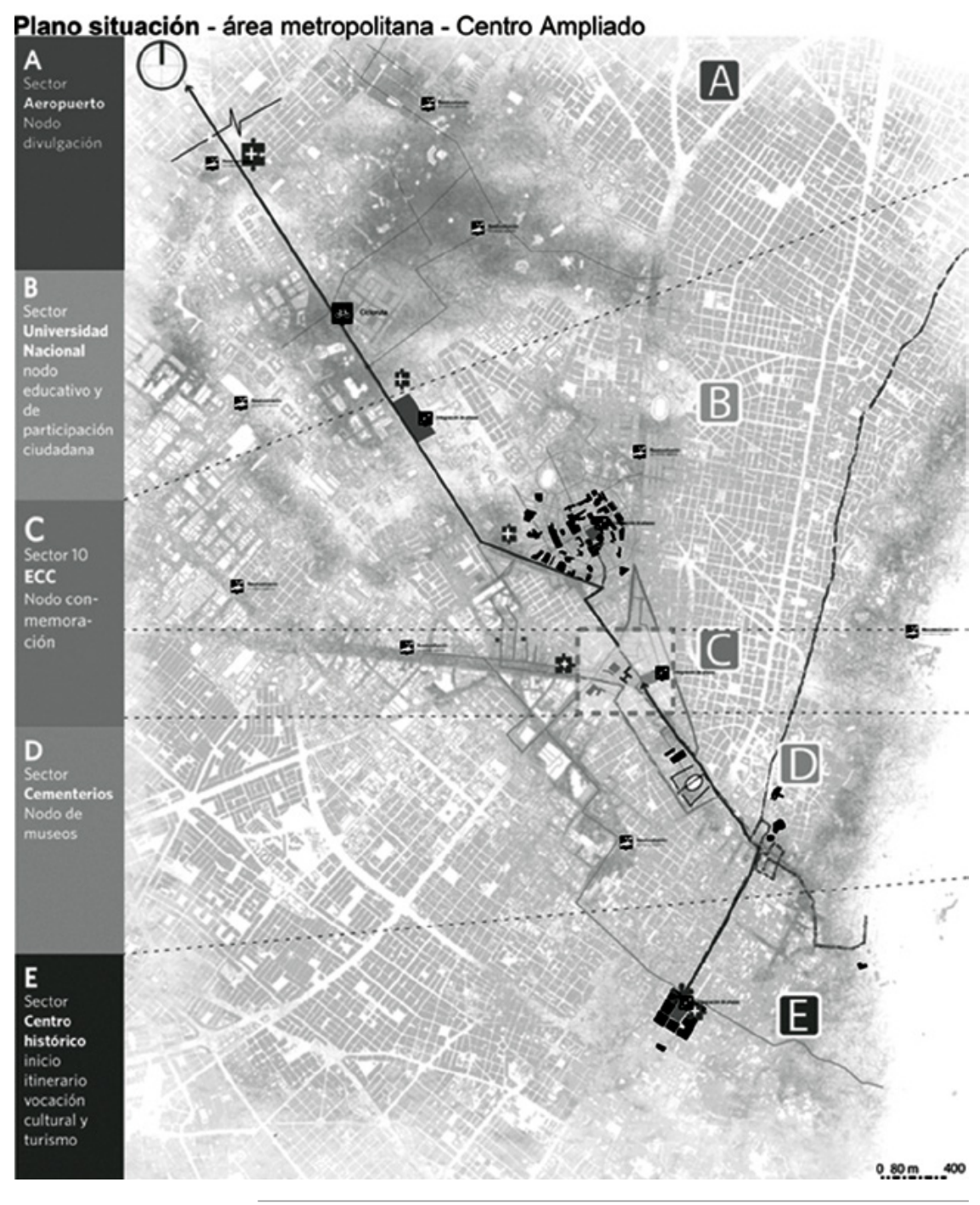

Figura 8. Escala de actuación metropolitana y localización sector $C$, correspondiente a la escala de actuación micro. 
Desde la forma y la condición del símbolo en el lugar se concluye con la última característica.

\section{3) El signo relacionado con el paisaje}

La característica de lugar como elemento de la red digital (Echeverría, 2009), y de escala más cercana para reconocer las reparaciones espirituales, como el duelo (Jelin, 2002), constituye el ambiente referente a la identidad, elemento propicio para consolidar el signo.

Este aspecto valida la premisa de carácter simbólico en el edificio y su situación urbana, en torno a las actividades conmemorativas y de orden visual, de forma que los materiales en la arquitectura se abordan desde la reinterpretación de las existencias y de los códigos presentes en los instrumentos de investigación (Puentes, 2018).

La materialidad se perfiló en la investigación hacia la evocación de las regiones y la representación de los tejidos, las cuales se expresaron en el sistema de fachadas, la estructura del escenario y el espacio colectivo (Puentes, 2018).

El hito y la altura como signo se emplearon para conformar el elemento de jerarquía, el cual se vincula en su situación o implantación para integrar el recinto, y se conforma para vincular los otros fragmentos de ciudad, como las plazoletas o áreas del contexto inmediato limitado por vías principales.

La forma del edificio representa abstractamente el conflicto, variable y diverso, por lo cual se ha plasmado con aspecto irregular (edificio blanco en la figura 9). 


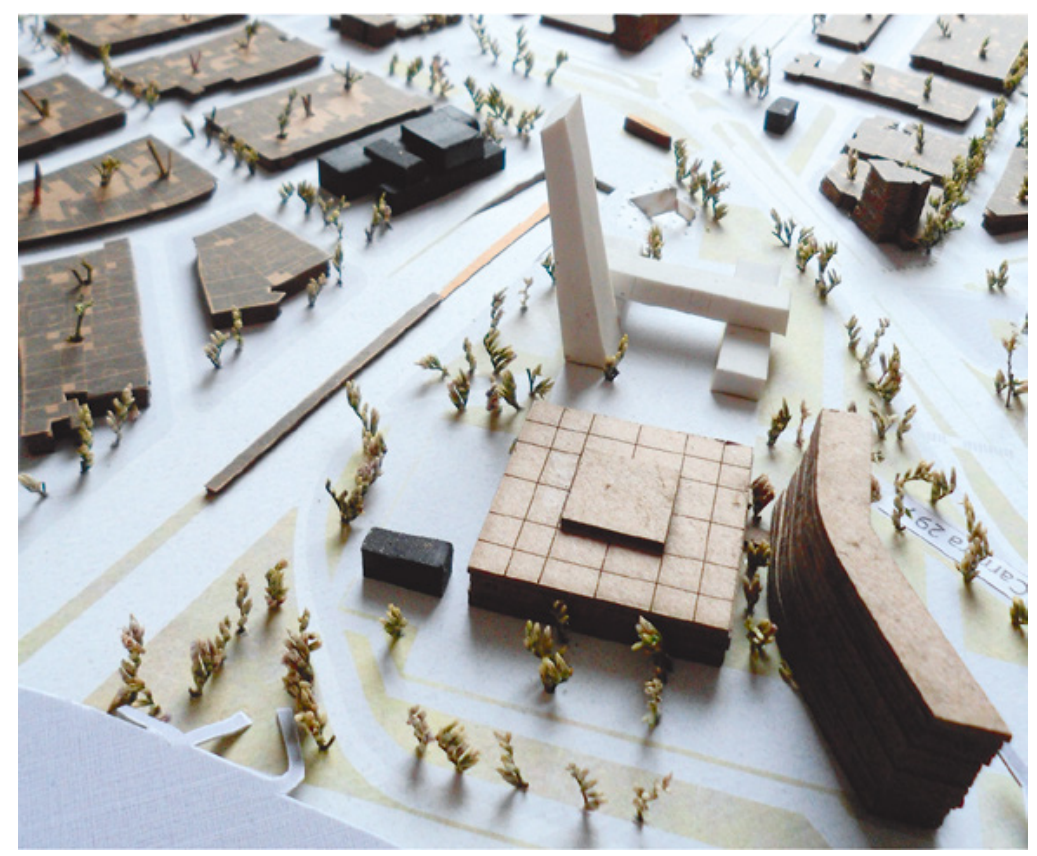

Figura 9. Escala micro, perspectiva urbana puente vehicular carrera 30 y CAD. Hipótesis proyectual del artefacto memorial, propuesta hacia la valoración de las categorías de la memoria en la ciudad de Bogotá.

Fuente: Autor.

Las preexistencias, como la escultura del Ala Solar, el Centro Administrativo Distrital y el Concejo de Bogotá (ver figura 1 para la ubicación geográfica en escala micro), poseen cualidades para recoger los elementos del entorno y hechos para narrar la actividad (sea esta administrativa, política o conmemorativa), por lo cual la relación de forma y contenido simbólico, en el contacto visual con el memorial y con la calle, provee significado a lo cotidiano.

Es de este modo como se materializa la memoria en un escenario y en un espacio colectivo de soporte, como resultado de la hipótesis proyectual, en la cual se postula al artefacto como un elemento para señalar el paisaje, signo de la identidad urbana y de calidad de vida (patrimonio y cultura, ver figura 6 y figura 9), de manera que la conceptualización de los espacios favorezca el carácter conmemorativo.

Las conclusiones que se presentan a continuación apuntan hacia la valoración de los aspectos cualitativos y proyectuales plasmados anteriormente, los cuales permiten concretar elementos comunes, cuando un hecho (para este caso de la guerra) es diverso y atañe a diferentes poblaciones. 


\section{Conclusiones}

Con respecto a las intervenciones y la forma de abordar e integrar diversos aspectos de carácter intangible en el ámbito del paisaje y la memoria para el proceso de diseño, se extractaron los elementos claves de la investigación teórica y proyectual en tres categorías principales, a partir de los resultados presentados anteriormente, como recomendaciones que se podrían aplicar para la planeación de proyectos de carácter patrimonial o conmemorativo.

\section{1) El usuario, el habitante y actor principal de la narrativa del lugar}

-El escenario, sea para el espacio público o para el memorial que favorezca el diálogo, es fundamental en el proceso de reparación simbólica, de reflexión y de pedagogía.

-Los elementos de calidad de vida en los procesos para la proyección de lo simbólico, vistos desde la identidad, aportan condiciones para definir el territorio y el lugar de la memoria desde la gestión y las decisiones de diseño; constituyen, en palabras de Roldán (2009, p. 79), “la apropiación, la cual se entrelaza con la identidad como acto de imaginación a través de la representación de los artefactos".

\section{2) Las dinámicas de los territorios}

-La medición de la memoria en el ámbito de la calidad de vida mediante la red en la región (local y nacional) se concreta a través de los lugares de memoria.

-En los territorios de conmemoración es importante vincular en el diseño el eje espiritual, de sanación o reparación, para generar arraigo en el lugar.

-La narración de la memoria, a través de la museografía y los objetos digitales, identificados por Echeverría (2009, p. 563) como "el texto, páginas web, la imagen, el audio", entre otros dispositivos útiles para exposiciones en el espacio público y los lugares de memoria, tiene el potencial de colaborar en transferir la información y construir espacios para la participación y reconocimiento del territorio colombiano, además de proveer accesibilidad y descentralización de las actividades por toda la región.

-Uno de los elementos clave, al abordar la memoria en el espacio y el memorial, es concentrar un hecho común reconocido por los usuarios y la población, el cual servirá de base para consolidar un eje narrativo en las diferentes escalas de actuación. 


\section{3) El signo relacionado con el paisaje}

-El artefacto (memorial), como uno de los signos de la memoria colectiva, puede sugerir diferentes lecturas, por esta razón es importante consolidar nodos e integrarlos en red, sea esta física y/o digital.

-Los elementos estéticos, vinculados a la identidad para generar el signo, derivan de las representaciones simbólicas del imaginario del lugar; estos se identifican a partir del trabajo de campo y los análisis precedentes a la intervención del lugar.

-Enfocar elementos de intangibilidad y dimensión emocional en los espacios de memoria, junto a los hitos naturales y artificiales, para conformar una unidad, es un ejercicio que permite obtener un panorama mucho más legible del espacio.

A manera de cierre, la integración de los tres aspectos mencionados anteriormente, con la sumatoria de la cultura, la reflexión y la accesibilidad, constituye una propuesta que permite contribuir a la conformación de la identidad, descifrar qué hecho histórico se narrará en la memoria del país, y de esta manera dar significado a espacios con carácter patrimonial o conmemorativo.

\section{REFERENCIAS BIBLIOGRÁFICAS}

Augé, M. (2000). Los «no lugares», espacios del anonimato. Una antropología de la sobremodernidad. España: Gedisa Editorial.

Cabrera, L. (2013). El derecho a la memoria y su protección jurídica: avance de investigación 1. Pensamiento Jurídico, (36). Recuperado de: https://revistas.unal.edu.co/index.php/peju/article/view/40321

Centro Nacional de Memoria Histórica (2018). En Colombia 82.998 personas fueron desaparecidas forzadamente. Recuperado de: http://centrodememoriahistorica.gov.co/en-colombia-82-998-personasfueron-desaparecidas-forzadamente/

Centro Nacional de Memoria Histórica (2015). Una nación desplazada: informe nacional del desplazamiento forzado en Colombia. Bogotá: CNMH - UARIV. Recuperado de: http://www. centrodememoriahistorica.gov.co/descargas/informes2015/nacion-desplazada/una-naciondesplazada.pdf 
Congreso de Colombia (10 de junio de 2011). Por la cual se dictan medidas de atención, asistencia y reparación integral a las víctimas del conflicto armado interno y se dictan otras disposiciones. [Ley 1448 de 2011]. DO: 48096. Recuperado de: http://www.alcaldiabogota.gov.co/sisjur/normas/ Norma1.jsp?i=43043

Echeverría, J. (2009). Cultura digital y memoria en red. Arbor, 185(737), 559-567. doi: http://dx.doi. org/10.3989/arbor.2009.i737.313

El Tiempo (26 de septiembre de 2016). "Hoy se firma el final el final de 52 años de guerra". El Tiempo. Recuperado de: http://www.eltiempo.com/politica/proceso-de-paz/firma-de-la-paz-en-colombia-2016-31456

Indepaz (2015). Memoria, paz y reconciliación. El centro en imágenes. Bogotá: Centro de Memoria, Paz y Reconciliación. Recuperado de: http://www.indepaz.org.co/wp-content/uploads/2017/09/ Memoria-paz-y-reconciliaci\%C3\%B3n.pdf

Instituto Distrital de Patrimonio (2015). Informe de empalme. Recuperado de: https://www. culturarecreacionydeporte.gov.co/sites/default/files/documento_empalme_idpc_04_11_15.pdf

Jelin, E. (2002). Los trabajos de la memoria. Madrid: Siglo XXI de España Editores. Social Science Research Council.

Leva, G. (2005). Indicadores de calidad de vida urbana. Teoría y metodología. Recuperado de: http:// hm.unq.edu.ar/archivos_hm/GL_ICVU.pdf

Observatorio Distrital de Víctimas y Oficina de la Alta Consejería para los Derechos de las Víctimas, la Paz y la Reconciliación. (2017). Víctimas de Bogotá. Recuperado de: http://observatorio.victimasbogota. gov.co/sites/default/files/documentos/PRISMA\%201.pdf

Oficina de Naciones Unidas para la coordinación de Asuntos Humanitarios (OCHA, 2015). Infografía. Brechasen la calidad de vida delos desplazados en Colombia. Recuperado de: https://www.humanitarianresponse.info/ en/operations/colombia/infographic/brechas-en-la-calidad-de-vida-de-los-desplazados-en-colombia

Piñeiro, J. G. (2010). Aproximación a la formulación del diagnóstico territorial. Lurralde: Investigación y espacio, (33), 209-232. 
Presidencia de la República de Colombia (20 de diciembre de 2011). Por el cual se establece la estructura del Centro de Memoria Histórica. [Decreto 4803 de 2011]. DO: 48289. Recuperado de: http://www. alcaldiabogota.gov.co/sisjur/normas/Norma1.jsp?i=45078

Puentes, S. (2018). Escenario de Construcción Colectiva en la Red de la Memoria del Centro Ampliado del Paisaje de Bogotá. [Trabajo de grado, Universitaria Agustiniana]. Recuperado de: http:// repositorio.uniagustiniana.edu.co/handle/123456789/543

Red Colombiana de Lugares de Memoria (2018). Lugares de Memoria. Recuperado el 1 de marzo de 2018 en: http://redmemoriacolombia.org/site/

Roldán, A. C. (2009). Algunas observaciones teóricas al territorio y la memoria: la dialéctica de la producción (creación) social del espacio. Revista Geográfica, (145), 73-88.

Secretaría Jurídica Distrital de la Alcaldía Mayor de Bogotá D.C. (31 de diciembre de 2014). Por el cual se adopta el Proyecto de Diseño Urbano Eje de la Paz y la Memoria, que integra diferentes Conjuntos Monumentales de Espacio Público en la ciudad de Bogotá D.C., y se dictan otras disposiciones. [Decreto 632 de 2014]. Recuperado de: http://www.alcaldiabogota.gov.co/sisjur/normas/Norma1. jsp?i=60318

Souroujon, G. (2011). Reflexiones en torno a la relación entre memoria, identidad e imaginación. Andamios, 8(17), 233-257. Recuperado de: http://www.scielo.org.mx/scielo.php?script=sci_arttext\&pid=S1870 $-00632011000300011 \& \operatorname{lng}=\mathrm{es} \& \ln \mathrm{l}=\mathrm{es}$.

Toledo, M. (2012). Sobre la construcción identitaria. Atenea (Concepción), (506), 43-56. https://dx.doi. org/10.4067/S0718-04622012000200004

Unidad para las victimas (2018). RUV. Registro único de Víctimas. Recuperado el 9 de abril de 2018 en https://www.unidadvictimas.gov.co/es/registro-unico-de-victimas-ruv/37394

Velásquez, C. (2011). El museo memorial: un nuevo espécimen entre los museos de historia. Intervención (Méx. DF), 2(3), 26-31. 